\title{
LA BARAJA DEL JOKER: FACTORES DESENCADENANTES DE LA CONDUCTA AGRESIVA EN EL PACIENTE CON AFECCIÓN PSEUDOBULBAR
}

\section{The Joker's deck: factors triggering the aggressive behavior on a patient with a pseudobulbar affect}

\author{
Estefany VIANA-VIVANCO; Andrés Alejandro FLORES-PEREIRA; Alberto ALVAREZ-BAEZA; Reinhard \\ JANSSEN-AGUILAR; Nina I. MÉNDEZ-DOMÍNGUEZ \\ Universidad Marista de Mérida (México). \\ e-mail: nmendez@marista.edu.mx
}

Fecha de recepción: 30 de octubre de 2019

Fecha de aceptación: 2 de diciembre de 2019

Fecha de publicación: 15 de septiembre de 2020

\begin{abstract}
Resumen
La trama del Joker nos muestra el surgimiento del antihéroe a partir de Arthur, un individuo con un trastorno llamado afectación pseudobulbar, con antecedentes de abandono y abuso físico severo y maltrato con probable traumatismo encefálico, que vive sin vínculos sociales, carente de afecto, como cuidador primario de una familiar dependiente enteramente de él; el protagonista se mueve en un ambiente adverso, hostil en el que la sociedad que espera que él se comporte como si no tuviera ningún trastorno. un comportamiento normal". Sin recursos y rechazado por su medio, resulta colateralmente afectado por la reducción de presupuesto para terapia y medicamentos. En un momento decisivo, convergen los diferentes detonantes de sus manifestaciones psicopatológicas que dan paso a la transformación del joker y paradójicamente lo llevarán a ser aceptado socialmente. Joker nos invita a reflexionar sobre la importancia de a los programas de salud mental, los valores humanos, la cultura de la Paz y el buen trato como pilares fundamentales para promover la salud mental en la sociedad. El presente artículo tiene por objetivo el revisitar la fisiopatología de la afección pseudobulbar y establecer los aspectos clínicos, familiares, ambientales y socioculturales potencialmente asociados a la conducta destructiva de Joker.

Palabras clave: afección pseudobulbar; salud mental; psicopatología; abuso físico.
\end{abstract}

Rev. Med. Cine. 2020; 16(3), 165-174 Ediciones Universidad de Salamanca / @@ J. Med. Mov., 2020; 16 (3), 165-174 


\begin{abstract}
The plot of the Joker shows us the emergence of the antihero from Arthur, an individual with a pseudobulbar disorder, a history of abandonment and severe physical abuse and abuse with probable brain trauma, living without social ties, lacking affection, as a primary caregiver of a relative dependent entirely on him; the protagonist moves in an adverse, hostile environment in which the society that expects him to behave as if he had no disorder. normal behavior. "Without resources and rejected by its means, it is collaterally affected by the reduction of budget for therapy and medications. At a decisive moment, the different triggers of his psychopathological manifestations converge that give way to the transformation of the joker and paradoxically lead him to be socially accepted. Joker invites us to reflect on the importance of mental health programs, human values, the culture of Peace and good treatment as fundamental pillars to promote mental health in society. This article aims to revisit the pathophysiology of the pseudobulbar condition and establish the clinical, family, environmental and sociocultural aspects potentially associated with Joker's destructive behavior.
\end{abstract}

Key words: pseudobulbar affect; mental health; physchopathology; physical abuse.

\section{Ficha técnica}

Título: Joker.

País: Estados Unidos, Canadá.

Año: 2019.

Director: Todd Phillips.

Música: Hildur Guðnadóttir.

Fotografía: Lawrence Sher.

Montaje: Jeff Groth.

Guion: Todd Phillips, Scott Silver, Bob Kane, Bill Finger, Jerry Robinson.

Intérpretes: Joaquin Phoenix, Robert De Niro, Zazie Beetz, Frances Conroy, Brett Cullen, Shea Whigham, Bill Camp, Glenn Fleshler, Leigh Gill, Josh Pais, ...

Color: color.

Duración: 122minutos.

Género: crimen, drama, suspenso.

Sinopsis: «Arthur Fleck (Phoenix) vive en Gotham con su madre, y su única motivación en la vida es hacer reír a la gente. Actúa haciendo de payaso en pequeños trabajos, pero tiene problemas mentales que hacen que la gente le vea como un bicho raro. Su gran sueño es actuar como cómico delante del público, pero una serie de trágicos acontecimientos le hará ir incrementando su ira contra una sociedad que le ignora» (Filmaffinity).
Productora: BRON Studios, Creative Wealth Media Finance, DC Comics, DC Entertainment, Joint Effort, Village Roadshow Pictures, Warner Bros.

Premios: Festival de cine de Venecia: Ganadora del premio Fanheart, Graffetta d'Oro a la mejor película (2019), ganadora del premio Golden Lion a la mejor película (2019), ganadora del premio Soundtrack Stars Award a la mejor banda sonora (2019). Premios Golden Trailer: Nominada Golden Trailer al mejor teaser (2019).

Enlaces: https://www.imdb.com/title/tt7286456/ https://www.filmaffinity.com/es/film520214. html

\section{Trailer}

¿Qué sucedería si un día nos fuese imposible expresar nuestras emociones del modo en el que queremos? ¿Qué opinaría la sociedad si nuestro lenguaje no verbal fuese contradictorio con el momento que vivimos?

Ese es precisamente el sentir que define a una persona con afección pseudobulbar, al saber que, aun estando consciente de su condición, la gente espera que se comporte como si ésta no existiera.

Rev. Med. Cine. 2020; 16(3), 165-174 Ediciones Universidad de Salamanca / @@@ J. Med. Mov., 2020; 16 (3), 165-174 


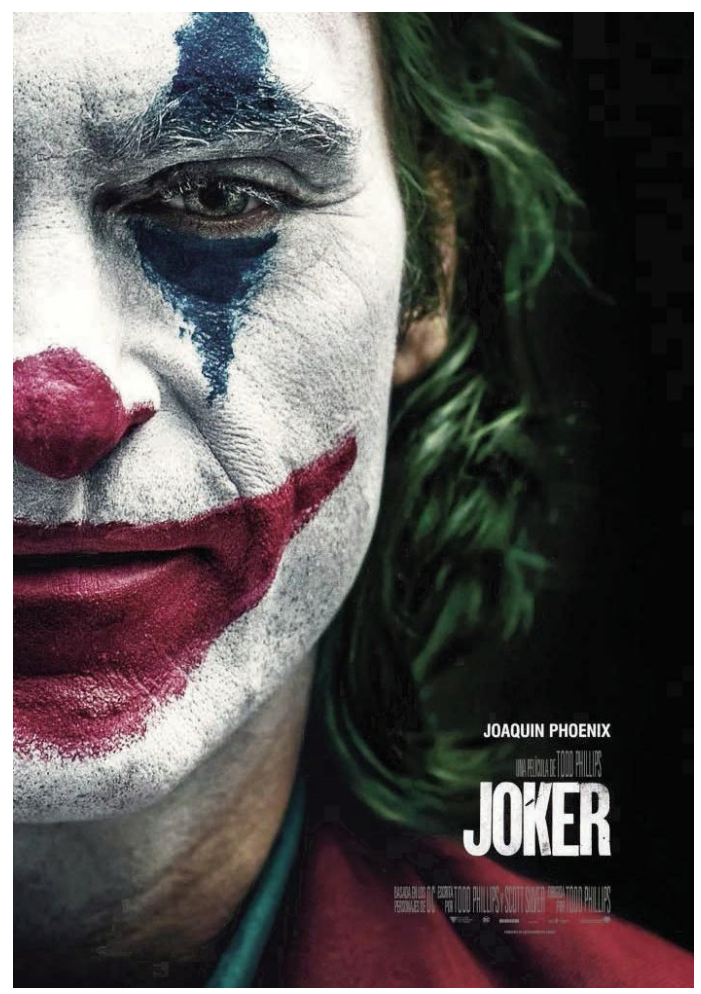

Cartel español.

Quizás algún lector al observar al Joker pudiera encontrar similitudes entre éste y el comportamiento de Arlequín, protagonista de la ópera Payasos de Ruggero Leoncavallo. Sin embargo, dicha analogía no podría ser más imprecisa, dado que la naturaleza de Arlequín le brindaba tanto control sobre su expresión y comportamiento, que le permitía voluntariamente actuar del modo opuesto a sus sentimientos y emociones.

Por el contrario, Joker carece totalmente de control, es ajeno a su propia expresión, incapaz de controlar sus deseoso de modificar su entorno mediato o inmediato. Si bien es cierto que tanto el multiverso de historias entrelazadas de los personajes del universo DC y el verismo italiano decimonónico comparten ambos una tendencia dramática por representar la miseria en la que se sumergen las clases sociales mediante la tragedia derivada de sus propios actos violentos, Arlequín fue capaz de encontrar el arrepentimiento, mientras Joker carece genuinamente de él.

Quizás considerando esta ausencia de arrepentimiento en Joker, nos inclinemos a pensar que Joker es un villano genuino. Sin embargo, en las historias fantásticas que inundan nuestra época, un villano es la némesis del superhéroe, aquel que se contrapone a sus planes altruistas, lo cual demuestra que Joker no cumple con esa característica distintiva de los villanos.

Dado que, en la trama de Joker, el héroe resulta dañado colateralmente y ante la duda de si fue el azar versus la desaventurada convergencia de una enfermedad exacerbada por condiciones psicosociales adversas, nos planteamos para el presente artículo el objetivo de establecer los aspectos clínicos, familiares, ambientales y socioculturales potencialmente asociados a la conducta destructiva del protagonista.

Aspectos clínicos y manifestaciones características

De acuerdo con el diccionario MerriamWebster, Joker se define como una persona insignificante, desagradable o incompetente $y$, es justamente de ese modo como el director se dispuso a representar al personaje caracterizado por Joaquim Phoenix. Arthur (Joker) de acuerdo con las manifestaciones caracterizadas en la película por Joker, éste sufre de un trastorno conocido como afección pseudobulbar (ASB).

La ASB genera un cuadro que se caracteriza por la presencia de una expresión emocional exagerada, inapropiada en relación con el contexto de una situación que no amerita dicho comportamiento. En esta condición encontramos una desproporción en la expresión emocional del paciente. Los estallidos y exacerbaciones del ASB pueden causar vergüenza, ansiedad y depresión, con frecuencia afectan la calidad de vida relacionada con la salud ${ }^{1}$ (Foto 1 ).

Rev. Med. Cine. 2020; 16(3), 165-174 Ediciones Universidad de Salamanca / @®@ J. Med. Mov., 2020; 16 (3), $165-174$ 
Esta forma de expresión emocional desordenada, caracterizada por estallidos de risa o llanto involuntario, incontrolable y estereotipado, ha sido reconocida desde fines del siglo XIX. Se pueden encontrar numerosas referencias a estas condiciones (Zwangslachen y Zwangsweinen señaladas por neurólogos alemanes y las espasmodiques rire et pleurer descritas por los franceses) en los escritos de Oppenheim, von Monakow y Wilson. El término incontinencia emocional aplicado por los psiquiatras puede ser exacto, pero es a la vez peyorativo. La risa o el llanto descontextualizados siempre tienen una base patológica en el cerebro, ya sea difusa o focal. Ha habido informes de risa espasmódica después de un infarto estriatocapsular unilateral y se han reportado casos ocasionales después de un infarto pontino unilateral o malformación arteriovenosa, pero estos no fueron verificados ${ }^{2,3}$.

En la más mínima provocación y, a veces, sin razón aparente, el paciente con ASB se ve envuelto en un estereotipo de risa que puede durar por

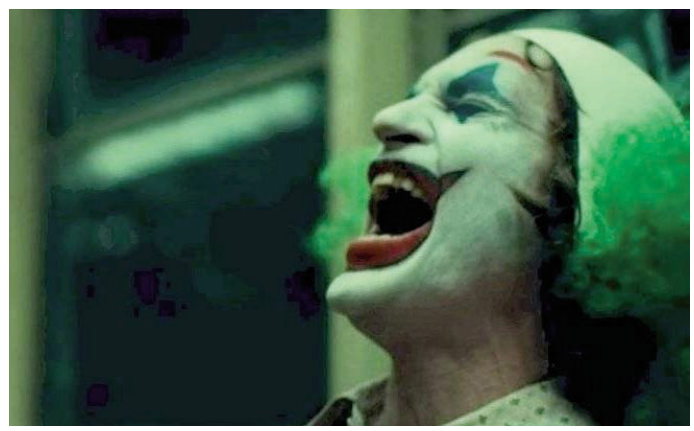

momentos o hasta muchos minutos, hasta el punto de agotamiento. La severidad del despliegue emocional y la facilidad con que se provoca no se corresponde con la severidad de la parálisis motora pseudobulbar o con una exageración de los reflejos tendinosos faciales y maseteros («jaw jerk»), por lo que difieren etiológicamente 4 .

Típicamente, el paciente con afección pseudobulbar expresa risa o llanto incoercible de manera impredecible e involuntaria, tal como observamos en el Joker, quien incluso viajaba con una tarjeta para hacerle saber a las demás personas que padecía un trastorno mental que lo orillaba a un comportamiento inadecuado. La ASB es una condición socialmente debilitante que puede aparecer dentro de una variedad de enfermedades neurológicas. Es también posible identificar estas características en pacientes diagnosticados con esclerosis lateral amiotrófica, trastornos extrapiramidales y cerebelosos, esclerosis múltiple, lesión cerebral traumática, enfermedad de Alzheimer, accidente cerebrovascular y tumores cerebrales ${ }^{5}$.

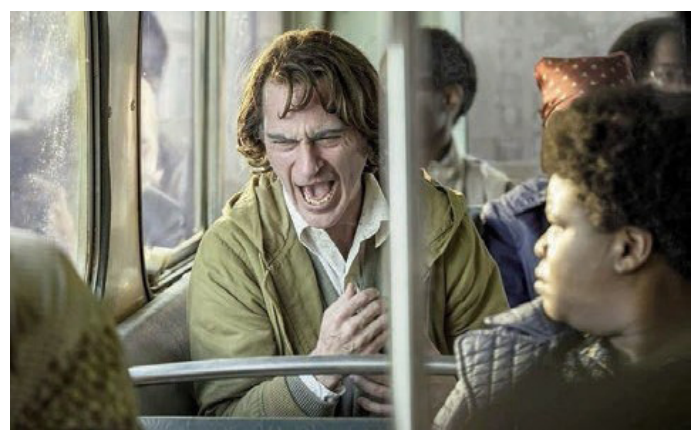

Foto 1. Joker carece totalmente de control, incapaz de controlar su expresión.

Etiología y génesis de la afección pseudobulbar

Aunque la fisio-patogenia de esta afección no está clara en la mayoría de los casos, diversos autores coinciden en que puede asociarse con lesiones craneoencefálicas con el desarrollo de la patología (tal como sucedió al Joker), pero algunos otros han identificado además los factores determinantes previos, entre los que se encuentra los rasgos de personalidad premórbida, el grado de afección post infarto, la historia personal de depresión, etc ${ }^{6}$.

Otros autores han descrito que las lesiones que provocan ASB suelen ser subcorticales y de localización frontal, sin encontrar mayor 
susceptibilidad referente al hemisferio involucrado. Adicionalmente, se han relacionado los hallazgos imagenológicos mediante la tomografía axial computarizada, los cuales incluyen lesiones corticales y subcorticales encontrando mayor frecuencia de ASB en las lesiones hemisféricas temporales y frontales, con predominio izquierdo. Otra teoría apunta a que la génesis de la ASB parecería ser consecuencia de la afección cerebelosa o de las vías cerebro-ponto-cerebelosas ${ }^{1,4,7}$.

Aunque la ASB es considerada una condición de reciente identificación, es de acuerdo con la evidencia documental relacionada, muy antigua. La ASB puede rastrearse en registros que datan del siglo XIX. Se ha encontrado que la ASB está relacionada con una mayor prevalencia de trastornos psiquiátricos diagnosticables y que entre el $30 \%$ a $35 \%$ de los pacientes con esta condición padecen depresión ${ }^{4}$.

La terminología para describir a la ASB ha sido variada y, por lo tanto, resulta prácticamente imposible de homologar. Si bien los términos como expresión emocional involuntaria, labilidad emocional, desregulación emocional, risa patológica con Ilanto, incontinencia emocional y emocionalismo han sido empleados en este contexto, su empleo ha sido arbitrario ${ }^{1,2}$. Todo lo anteriormente mencionado contribuye al infra diagnóstico de esta enfermedad, y el manejo subóptimo de la misma.

\section{Diagnóstico de la afección pseubulbar}

A lo largo del tiempo la metodología utilizada para el diagnóstico de esta condición ha ido cambiando. En los registros sobre este tema que proceden de la década de 1980 , se menciona que el diagnóstico quedaba a criterio clínico del examinador. A partir de la década de 1990 , se han integrado distintas escalas diagnósticas objetivas $\mathrm{y}$, sobre todo, reproducibles. Posteriormente, se desarrolló y validó una escala diagnóstica para ASB en pacientes con accidente cerebro vascular agudo denominada PLACS, se validó también la escala Center for Neurologic Study-Lability Scale (CNS-LS) para ELA, cabe mencionar que esta última escala fue validada posteriormente para el diagnóstico de la esclerosis múltiple. Otra escala que ha sido utilizada en la mayoría de los trabajos realizados sobre esta afección fue el Inventario de depresión de Beck, su aplicación permite delimitar con firmeza la existencia o no de una afección que puede asemejarse o confundirse con la ASB. En 2011, nuevos criterios fueron propuestos por Miller, Pratt y Shiffer ${ }^{8}$ (Tabla 1).

Tabla 1. Criterios diagnósticos de la afección pseudobulbar propuestos por Miller, Pratt y Schiffer, $2011^{8}$.

\section{Criterios esenciales}

El paciente experimenta episodios de expresión emocional involuntaria o exagerada que resultan de un trastorno cerebral, incluidos episodios de risa, llanto o manifestaciones emocionales relacionadas.

Los episodios representan un cambio en la reactividad emocional habitual del paciente, son exagerados o incongruentes con el estado emocional subjetivo del paciente y son independientes o exceden el estímulo que provoca.

Los episodios causan angustia o discapacidad clínicamente significativas en el funcionamiento social u ocupacional. Los síntomas no pueden atribuirse a otro trastorno neurológico o psiquiátrico ni a los efectos de una sustancia.

\section{Criterios de apoyo}

El paciente puede experimentar cambios autonómicos acompañantes (p. Ej., Enrojecimiento de la cara) y signos pseudobulbar (p. Ej., Acentuación de la sonrisa a expensas de la mandíbula, reflejo nauseoso exagerado, debilidad de la lengua, disartria y disfagia).

Los pacientes pueden exhibir propensión a la ira. 


\section{Tratamiento integral de la afección pseudobulbar}

El manejo de un paciente con ASB dista mucho de ser simple, pues si bien requiere manejo farmacológico, también involucra la psicoterapia, de un ambiente psicosocial propicio, seguimiento y apoyo familiar y social.

El tratamiento de la ASB en años recientes ha implicado principalmente el uso de medicamentos que modulan la neurotransmisión serotoninérgica o glutamatérgica. Las terapias serotoninérgicas como la amitriptilina y la fluoxetina pueden ejercer efectos al aumentar la serotonina en la sinapsis, y el dextrometorfano puede actuar a través de efectos antiglutamatérgicos en los receptores NMDA y los receptores $\sigma-1$. Sin embargo, es importante tener en cuenta que los serotoninérgicos y los antidepresivos tricíclicos tienen afinidad con una amplia variedad de tipos de receptores, incluidos los receptores $\sigma-1$, aunque las afinidades de unión en el receptor $\sigma-1$ son menores para la fluoxetina y la amitriptilina que para el dextrometorfano. Del mismo modo, el perfil de unión del dextrometorfano no se limita a los receptores $\sigma-1$ o NMDA, sino que también incluye afinidades por el transportador 5-HT y los receptores noradrenérgicos $\alpha-2$ (aunque inferiores a los informados para fluoxetina o amitriptilina) $)^{7,8}$.

En algunos pacientes, la neuropatología puede estar limitada a sustratos involucrados principalmente en la expresión motora de la emoción, mientras que, en otros pacientes, el daño puede extenderse a áreas y sistemas neuronales involucrados en un estado emocional sostenido (estado de ánimo), Tanto si los síntomas de ASB se acompañan de depresión, como si no lo hacen. Las vías neurales que se cree que están involucradas en la depresión son generalizadas, complejas e involucran múltiples sistemas de neurotransmisores ${ }^{3,4,4,10}$.

Aun cuando los antidepresivos por si solos han mostrado beneficios, los datos clínicos de apoyo a menudo se han derivado de un pequeño número de pacientes y medidas no validadas de la gravedad de la ASB. El dextrometorfano + quinidina, es el primer medicamento para ASB aprobado por la FDA (Administración de alimentos y medicamentos de los estados Unidos), al ser una terapia novedosa con acciones antiglutamatérgicas. A medida que la esperanza de vida se alarga y la configuración neurológica de la ASB se vuelve más común, se puede esperar que aumente la necesidad de tratamientos innovadores ${ }^{4}$.

La historia del Joker incluye la alusión a las condiciones económicas de los programas sociales de salud mental, mostrando como los altos mandos del gobierno de ciudad gótica gozaban de privilegios y excesivos lujos, la población en su mayoría experimentaba una desatención en los recursos de higiene y de atención a la salud y en particular, a la salud mental. Es posible identificar que si bien, el programa de psicoterapia y los fármacos que recibía Joker de los programas municipales no eran del todo óptimos ni de su agrado, al suspenderse este apoyo por motivos económicos, la estabilidad y bienestar del protagonista se vieron gravemente afectadas.

El acompañamiento, la terapia psicológica y el apoyo social y familiar son indispensables para lograr una socialización lo más sana posible, así como también para desarrollar medidas de afrontamiento de esta patología. Un ambiente familiar propicio, como en todo padecimiento neuropsiquiátrico, el apoyo social es fundamental para lograr la integración e inclusión de los pacientes en las distintas esferas de desempeño, como lo son las escolares y laborales ${ }^{9}$.

Desafortunadamente, la trama del joker revela que las condiciones familiares del protagonista no fueron menos que traumáticas, habiendo sido víctima de maltrato y un abuso severo, constante y prolongado por parte de su madre, quien lo creció como a su hijo único en un hogar uniparental de escasos recursos. Joker poseía un profundo vacío afectivo al considerarse rechazado por parte de un padre al que no conocía y ello se refleja en su necesidad por hallar la aprobación de personas como el conductor del programa televisivo, teniendo

Rev. Med. Cine. 2020; 16(3), 165-174 Ediciones Universidad de Salamanca / @@@ J. Med. Mov., 2020; 16 (3), $165-174$ 
incluso delirios con respecto a que aquel personaje le trataría como a un hijo. Pero la verdadera intención del conductor al solicitar la presencia del joker generó un estallido de frustración e ira, semejante en cierta medida a aquella respuesta que tuvo hacia su madre ante el descubrimiento del maltrato y las condiciones de su infancia. Todo ello dejó al protagonista aislado, frustrado, herido y rencoroso.

En el ambiente social y político de ciudad gótica se adivina el peligro que se vive en todo aquel lugar donde hay una total falta de valores y la ausencia de empatía y caridad de una sociedad hastiada de haber sido subyugada. En ese medio se adivina el rencor en sus pobladores, que se alimentan de los vicios, de la sed de venganza y la violencia se esparce como un virus entre los ciudadanos.

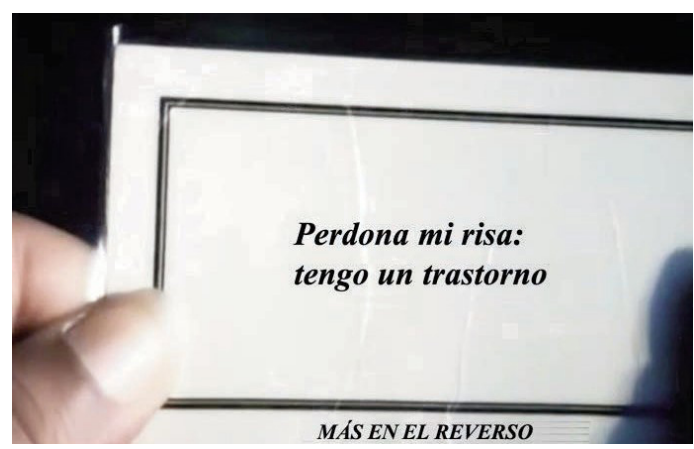

Foto 2. Joker viajaba con una tarjeta para hacerle saber a las demás personas que padece un trastorno mental que lo orillaba a un comportamiento inadecuado.

El riesgo de una enfermedad mental grave es generalmente mayor en las ciudades en comparación con las zonas rurales. Los estudios epidemiológicos han asociado el crecimiento y la vida en ciudades con un riesgo considerablemente mayor de esquizofrenia. Sin embargo, la correlación no es causalidad y vivir en la pobreza puede contribuir y resultar de los impedimentos asociados con la mala salud mental. El aislamiento social y la discriminación, así como la pobreza en el vecindario, contribuyen a la carga de salud mental, mientras que se sabe poco sobre las interacciones específicas entre tales factores y el entorno construido, pero además de todo ello, de la patología subyacente, de las carencias y la violencia experimentada en su niñez, además de habitar en un medio urbano hostil, en la vida de Joker se sumaron aún más elementos ${ }^{11-13}$ (Foto 2 ).

El latente síndrome del cuidador en la vida del Joker

El síndrome del cuidador es un estado de agotamiento físico y psíquico que se presenta en personas que desempeñan el rol de cuidador principal de una persona dependiente, mismos que presentan una mayor morbilidad de padecer trastornos psiquiátricos. En este sentido, Dueñas y colaboradores señalaron una mayor prevalencia de depresión, ansiedad y disfunción familiar severa en comparación con el grupo control de no cuidadores. Siguiendo la misma línea, un estado depresivo aunado a una condición psiquiátrica de base previa, podría ser un catalizador de un brote psicótico, por lo que coincidimos con Vargas y colaboradores quienes señalan una asociación entre los síntomas depresivos y los estados psicóticos. Al ser Joker el cuidador único de su madre discapacitada por un periodo tan prolongado, pueden identificarse los signos que sugieren que padecía el síndrome del cuidador. Tomando en cuenta que el síndrome del cuidador se asocia a mayores niveles de depresión y ansiedad y estos, con un riesgo incrementado de detonar y agudizar los brotes psicóticos, es coherente sugerir que, además de la coexistencia con los factores antes mencionados, favoreció las ideaciones delirantes del Joker ${ }^{14,15}$.

El estrés psicosocial como detonante inmediato en conducta agresiva del Joker

El estrés puede ser definido como la percepción de una amenaza ante la cual el organismo 
requiere de adaptaciones para asegurar la homeostasis y la supervivencia ${ }^{15}$. En la misma línea argumental, podemos entonces definir el estrés psicosocial como el estrés generado por situaciones sociales y/o emocionales conflictivas, que puede desencadenar como consecuencia diversas patologías y conductas en función de la predisposición genética, la experiencia del individuo y su ambiente cultural ${ }^{16}$.

Una emoción común en el estrés es el miedo, emoción que no requiere de cognición y que se regula mediante la amígdala del lóbulo temporal. El miedo se activa ante determinadas situaciones, teniendo un único fin: la supervivencia ${ }^{17}$. Gray propuso en 1987 un sistema de inhibición conductual modulado por el hipocampo y el septum, condición que le permite al individuo aumentar su estado de alerta y prepararse para hacerle frente al estímulo estresante ${ }^{18,19}$.

En la escena donde Joker asesina a tres asaltantes agredían, se puede apreciar en él aquella sonrisa patológica que precedió a la agresión, apareció. Durante la agresión, es posible que sintiera un profundo miedo y, en consecuencia, se detonara el sistema de inhibición conductual propuesto por Gray. Así mismo, Molina-Jiménez sugiere que el estrés psicosocial puede desencadenar una serie de conductas que pueden variar de persona en persona dependiendo de su contexto. Dado que Joker se encontraba regresando a casa luego de haber sido despedido, con toda la carga emocional, frustración y preocupación que ello le confería, el hecho de haber sido nuevamente atacado lo invadió con miedo y ante la ocasión y la oportunidad de venganza que le confería el poseer un arma cargada, Joker sin mucho que perder tomó la solución que encontró a su alcance, abriendo paso a la ira, a la violencia extrema, de modo que al defenderse de los asaltantes, estaba cobrando venganza no solo por la afrenta de aquellos, sino también de todos los que previamente le infringieron miedo y le causaron daño ${ }^{18,19}$ (Foto 3).

\section{Discusión}

Hemos analizado a lo largo de este manuscrito, cómo las condiciones de desventaja social, afectiva, económica y biológica convergieron en el personaje de un adulto con demasiada carga emocional que se enfrenta a la adversidad.

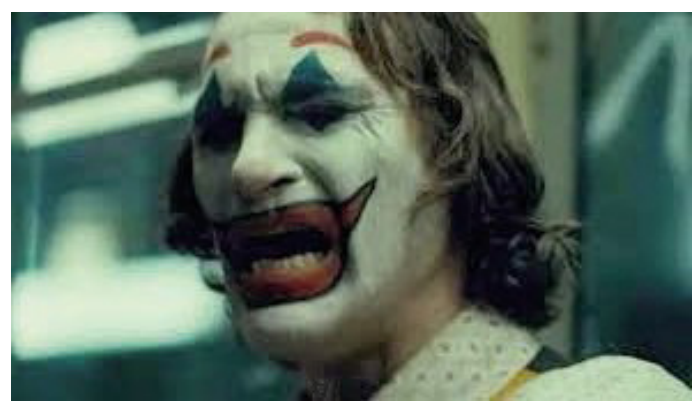

Foto 3. El Joker expresa risa y llanto al ser agredido por los asaltantes.

Si bien la convergencia de tantas situaciones adversas que atañen a una misma persona es más posible que probable, el filme nos hace reflexionar sobre los complejos problemas sociales y antropológicos que agravan los problemas neuropsiquiátricos cada vez más comunes, convirtiéndolos simultáneamente en víctimas y victimarios.

Se estima que pronto los problemas neuropsiquiátricos constituirán en breve la primera causa de ausentismo laboral, particularmente, la depresión mayor, por lo cual hemos de apostar por una formación médica más sensible a la comunicación, menos mecanizada ${ }^{20}$.

Tenemos como profesionales de la salud, el derecho y el deber de exigir a nuestras autoridades la justa destinación de recursos para la prevención y atención a los problemas de salud mental, pues no hay salud en ausencia de salud mental ${ }^{21,22}$.

Finalmente, consideramos que es urgente devolver a la educación integral desde el nivel básico, la cultura de la inclusión, de la empatía y los valores, que finalmente, son los que definirán en buena parte, el bienestar y la calidad de vida de las generaciones futuras. Ello sin duda 
abonaría a reducir el estrés psicosocial, permitiría una mejor inclusión de las personas afectadas por trastornos neuropsiquiátricos y permitiría prevenir que la historia del Joker, en un futuro cercano, se convierta más que en un cómic, en una basada en hechos reales.

\section{Referencias}

1. Ahmed A, Simmons Z. Pseudobulbar affect: prevalence and management. Ther Clin Risk Manag. 2013;9:483-9.

2. Quarracino C, Garreto NS, Arakaki T, Franco A, González L, Morera NB, et al. Frecuencia de afección seudobulbar en pacientes con esclerosis lateral amiotrófi$\mathrm{ca}$, enfermedad de Parkinson, esclerosis múltiple y accidente cerebrovascular. Neurol Arg. 2014;6(3):142-8.

3. Sauve WM. Recognizing and treating pseudobulbar affect. CNS Spectr. 2016;21(S1):34-44.

4. Chang YD, Davis MP, Smith J, Gutgsell T. Pseudobulbar Affect or Depression in Dementia? J Pain Symptom Manage. 2016;51(5):954-8.

5. Work SS, Colamonico JA, Bradley WG, Kaye RE. Pseudobulbar affect: an under-recognized and under-treated neurological disorder. Adv Ther. 2011;28(7):586-601.

6. Strowd RE, Cartwright MS, Okun MS, Haq I, Siddiqui MS. Pseudobulbar affect: prevalence and quality of life impact in movement disorders. J Neurol. 2010; 257(8):1382-7.

7. Crumpacker DW. Enhancing Approaches to the Identification and Management of Pseudobulbar Affect. J Clin Psychiatry. 2016;77(9):e1155.

8. Miller A, Pratt H, Schiffer RB. Pseudobulbar affect: the spectrum of clinical presentations, etiologies and treatments. Expert Rev Neurother. 2011;11(7):1077-88.

9. Demler TL. Introduction to pseudobulbar affect: setting the stage for recognition and familiarity with this challenging disorder. Am J Manag Care. 2017;23(18 Suppl):S339-s44.

10. Chen JJ. Pharmacotherapeutic management of pseudobulbar affect. Am J Manag Care. 2017;23(18 Suppl):S345-s50.
11. Allen J, Balfour R, Bell R, Marmot M. Social determinants of mental health. Int Rev Psychiatry. 2014;26(4):392-407.

12. Gruebner O, Rapp MA, Adli M, Kluge U, Galea S, Heinz A. Cities and Mental Health. Dtsch Arztebl Int. 2017;114(8):121-7.

13. Catala-Lopez F, Ridao M. Cost-effectiveness analyses in neuropsychiatry and mental health. Actas Esp Psiquiatr. 2017;45(3):127-36.

14. Gutiérrez-García A, Calvo MG. Social anxiety and threat-related interpretation of dynamic facial expressions: sensitivity and response bias. Pers Indiv Differ. 2017 Mar 1;107:10-6.

15. Vargas $T$, Ahmed AO, Strauss GP, Brandes CM, Walker EF, Buchanan RW, et al. The latent structure of depressive symptoms across clinical high risk and chronic phases of psychotic illness. Transl Psychiatry. 2019;9(1):229.

16. Mitra R, Jadhav S, McEwen BS, Vyas A, Chattarji $S$. Stress duration modulates the spatiotemporal patterns of spine formation in the basolateral amygdala. Proc Natl Acad Sci U S A. 2005;102(26):9371-6.

17. Goodman AM, Harnett NG, Wheelock MD, Hurst DR, Orem TR, Gossett EW et al. Anticipatory prefrontal cortex activity underlies stress-induced changes in Pavlovian fear conditioning. Neurolmage. 2018;174:237-47.

18. Dalgleish T. Cognitive approaches to posttraumatic stress disorder: the evolution of multirepresentational theorizing. Psychol Bull. 2004;130(2):228-60.

19. Xu M, Li Z, Ding C, Zhang J, Fan L, Diao L, et al. The Divergent Effects of Fear and Disgust on Inhibitory Control: An ERP Study. PLoS One. 2015;10(6):e0128932.

20. Johnston DA, Harvey SB, Glozier N, Calvo RA, Christensen $H$, Deady $M$. The relationship between depression symptoms, absenteeism and presenteeism. J Affect Disord. 2019 ;256:536-40.

21. Velásquez NR. Análisis de la relación entre la salud mental y el malestar humano en el trabajo. Equidad Desarro. 2017(29):161-78.

22. Danesh HB. Creating a culture of healing in multiethnic communities: an integrative approach to prevention and amelioration of violence-induced conditions. J Community Psychol. 2008;36(6):814-32.

Rev. Med. Cine. 2020; 16(3), 165-174 Ediciones Universidad de Salamanca / @®@ J. Med. Mov., 2020; 16 (3), $165-174$ 


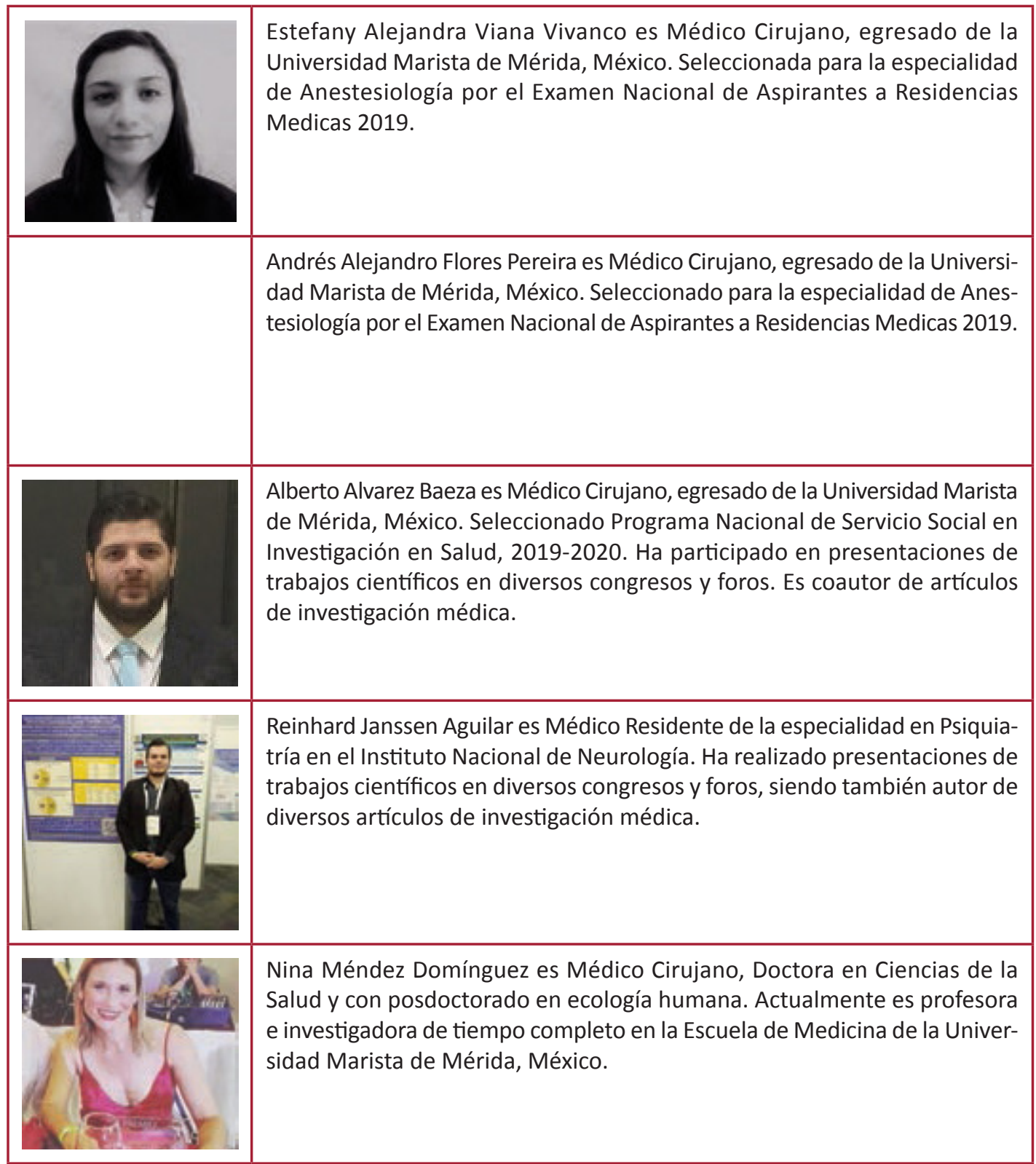

